\title{
Exponential tightness for Gaussian processes, with applications to some sequences of weighted means
}

\author{
Claudio Macci* $\quad$ Barbara Pacchiarotti ${ }^{\dagger}$
}

\begin{abstract}
Exponential tightness plays a crucial role in large deviations; in fact this condition is often required to obtain the large deviation principle for a sequence of random variables taking values on an infinite dimensional topological space. In this paper we present some conditions which yield the exponential tightness of a sequence of continuous Gaussian processes. Moreover we check these conditions for some sequences of weighted means.
\end{abstract}

Mathematics Subject Classification: 60F10, 60G15.

Keywords: covariance function, Hölder condition, large deviations, self-similar process.

\section{Introduction}

Large deviations give an asymptotic computation of small probabilities on an exponential scale (see e.g. [4] as a reference on this topic). We recall some basic definitions (see e.g. Section 1.2 in [4]). Throughout this paper a speed function is a sequence $\left\{v_{n}: n \geq 1\right\}$ such that $\lim _{n \rightarrow \infty} v_{n}=\infty$. A sequence of random variables $\left\{Z_{n}: n \geq 1\right\}$, taking values on a topological space $\mathcal{X}$, satisfies the large deviation principle (LDP for short) with rate function $I$ and speed function $v_{n}$ if the following conditions hold: the function $I: X \rightarrow[0, \infty]$ is lower semicontinuous,

$$
\liminf _{n \rightarrow \infty} \frac{1}{v_{n}} \log P\left(Z_{n} \in O\right) \geq-\inf _{x \in O} I(x)
$$

for all open sets $O$, and

$$
\limsup _{n \rightarrow \infty} \frac{1}{v_{n}} \log P\left(Z_{n} \in C\right) \leq-\inf _{x \in C} I(x)
$$

for all closed sets $C$. A rate function is said to be good if all its level sets $\{\{z \in Z: I(z) \leq \eta\}: \eta \geq 0\}$ are compact. Moreover $\left\{Z_{n}: n \geq 1\right\}$ is exponentially tight with respect to the speed function $v_{n}$ if, for all $b>0$, there exists a compact $K_{b} \subset \mathcal{X}$ such that

$$
\limsup _{n \rightarrow \infty} \frac{1}{v_{n}} \log P\left(Z_{n} \notin K_{b}\right) \leq-b .
$$

The concept of exponential tightness plays a crucial role in large deviations; in fact this condition is often required to establish that the large deviation principle holds for a sequence of random variables taking values on an infinite dimensional topological space. In this paper we refer to Conditions (i) and (ii) in Theorem 1.1 which yield the exponential tightness when the topological space $X$ is $C[0,1]$ equipped with the topology of the uniform convergence.

\footnotetext{
*Dipartimento di Matematica, Università di Roma Tor Vergata, Via della Ricerca Scientifica, I-00133 Rome, Italy. e-mail: macci@mat.uniroma2.it

${ }^{\dagger}$ Dipartimento di Matematica, Università di Roma Tor Vergata, Via della Ricerca Scientifica, I-00133 Rome, Italy. e-mail: pacchiar@mat.uniroma2.it
} 
Theorem 1.1 A sequence of random variables $\left\{Z_{n}: n \geq 1\right\}$, taking values on $C[0,1]$ (equipped with the topology of the uniform convergence), is exponentially tight with respect to the speed function $v_{n}$ if the two following conditions hold.

Condition (i) for increments: for some $n_{0} \in \mathbb{N}$ and $\alpha, \gamma, \kappa>0$,

$$
\mathbb{E}\left[\exp \left(\frac{\gamma v_{n}}{|t-s|^{\alpha}}\left|Z_{n}(t)-Z_{n}(s)\right|\right)\right] \leq \kappa^{v_{n}} \text { for all } t, s \in[0,1] \text { with } t \neq s .
$$

Condition (ii) for $t=0$ :

$$
\lim _{R \rightarrow \infty} \limsup _{n \rightarrow \infty} \frac{1}{v_{n}} \log P\left(\left|Z_{n}(0)\right|>R\right)=-\infty .
$$

A reference for this result is Theorem 3 in [17] which is proved as a consequence of Theorems 1 and 2 in the same reference; see also [5] (Theorem A.1 in [5] coincides with Theorem 1 in [17]) and [18].

The aim of this paper is to present Proposition 2.1 which yields the exponential tightness of a sequence of continuous Gaussian processes $\left\{Z_{n}: n \geq 1\right\}$ as a consequence of Theorem 1.1. Moreover these conditions are used to check the exponential tightness of some sequences $\left\{Z_{n}: n \geq 1\right\}$ defined by

$$
Z_{n}(t):=\frac{1}{A_{n}} \sum_{k=1}^{n} a_{k} X(k+t)(\text { for all } t \in[0,1]),
$$

where $\left\{a_{n}: n \geq 1\right\}$ is a sequence of positive numbers,

$$
A_{n}:=\sum_{k=1}^{n} a_{k}(\text { for all } n \geq 1)
$$

and $\{X(t): t \geq 1\}$ is a continuous real valued Gaussian process defined on a probability space $(\Omega, \mathcal{F}, P)$. Thus, in some sense, we give a completion of the results presented in [11].

The sequence $\left\{Z_{n}: n \geq 1\right\}$ defined by (1) is a sequence of weighted means and, as we see below, arithmetic and logarithmic means are allowed. Our interest in these weighted means is motivated by the strict analogy between the logarithmic means and the sequences of random measures which appear in the almost sure limit theorems. Here we recall the almost sure central limit theorem where, for $L(n):=\sum_{k=1}^{n} \frac{1}{k}$ (or, equivalently, $L(n):=\log n$ for $n \geq 2$ ), we have

$$
\left\{\frac{1}{L(n)} \sum_{k=1}^{n} \frac{1}{k} 1_{\left\{\frac{U_{1}+\cdots+U_{n}}{\sqrt{n}} \in \cdot\right\}}: n \geq 1\right\}
$$

for a sequence of i.i.d. centered random variables with unit variance $\left\{U_{n}: n \geq 1\right\}$. The almost sure central limit theorem was proved independently in [1], [9] and [16] under stronger moment assumptions; successive refinements appear in [10] and [13], in which only finite variance is required.

We remark that, if we consider the notation

$$
\mu(t):=\mathbb{E}[X(t)] \text { and } \varphi(t, s):=\operatorname{Cov}(X(t), X(s))
$$

for the mean and the covariance functions of the process $\{X(t): t \geq 1\}$, the mean and the covariance functions of the process $Z_{n}$ in (1) are

$$
m_{n}(t):=\mathbb{E}\left[Z_{n}(t)\right]=\frac{1}{A_{n}} \sum_{k=1}^{n} a_{k} \mu(k+t)
$$

and

$$
k_{n}(t, s):=\operatorname{Cov}\left(Z_{n}(t), Z_{n}(s)\right)=\frac{1}{A_{n}^{2}} \sum_{k, h=1}^{n} a_{h} a_{k} \varphi(k+t, h+s) .
$$


We consider different hypotheses on $\varphi(\cdot, \cdot)$ and $\left\{a_{n}: n \geq 1\right\}$; however we always assume that

$$
\lim _{n \rightarrow \infty} A_{n}=\infty
$$

More precisely we consider two conditions for the covariance function $\varphi(\cdot, \cdot)$, i.e. Conditions 3.1 and 4.1. Under Condition 3.1, we present two classes of coefficients $\left\{a_{n}: n \geq 1\right\}$ : for the first class we have

$$
\lim _{n \rightarrow \infty} \frac{\sum_{k=1}^{n} a_{k}^{2}}{A_{n}}=\ell, \text { for some } \ell \in(0, \infty),
$$

i.e. a generalization of the arithmetic means (i.e. $a_{n}=1$ for all $n \geq 1$, where (3) holds with $\ell=1$ ); for the second class we have

$$
C:=\sup \left\{n a_{n}: n \geq 1\right\} \in(0, \infty),
$$

i.e. a generalization of the logarithmic means (i.e. $a_{n}=\frac{1}{n}$ for all $n \geq 1$, where (4) holds with $C=1$ ). Under Condition 4.1 we consider only the second class of coefficients. Thus, if we combine the conditions on the covariance function $\varphi(\cdot, \cdot)$ and on the coefficients $\left\{a_{n}: n \geq 1\right\}$, we have three cases which can be related to Propositions 3.1, 3.2(i) and 3.3 in [11]. Those propositions in [11] show the existence of a covariance function $c(\cdot, \cdot)$ such that

$$
\lim _{n \rightarrow \infty} \frac{v_{n}}{A_{n}^{2}} \operatorname{Var}\left[\sum_{k=1}^{n} a_{k} \int_{0}^{1} X(k+t) d \theta(t)\right]=\int_{0}^{1} \int_{0}^{1} c(t, s) d \theta(t) d \theta(s)
$$

for all signed measures $\theta$ on $[0,1]$ with bounded variation. Here the limit (5) is briefly discussed (see Remarks 3.2 and 4.2) in terms of the conditions presented in this paper (for the covariance function $\varphi(\cdot, \cdot)$ and the coefficients $\left.\left\{a_{n}: n \geq 1\right\}\right)$. Finally, as in [11], we present some examples: in some cases the examples in this paper are more general than the ones in [11], and often they are formulated in terms of self-similar processes.

We conclude with the outline of the paper. In Section 2 we present Proposition 2.1 (which yields the exponential tightness of a sequence of continuous Gaussian processes $\left\{Z_{n}: n \geq 1\right\}$ ). Results for Conditions 3.1 and 4.1 (concerning the covariance function $\varphi(\cdot, \cdot)$ ) are presented in Sections 3 and 4, respectively. Some examples are presented in Section 5 (actually the first example appears in Section 3).

\section{Exponential tightness for continuous Gaussian processes}

The aim of this section is to present Proposition 2.1 which yields the exponential tightness of a sequence of continuous Gaussian process $\left\{Z_{n}: n \geq 1\right\}$ as a consequence of Theorem 1.1.

We start with the following lemma where we recall some estimates for Gaussian random variables (for the sake of completeness, we give some details on their proofs in the Appendix).

Lemma 2.1 Let $X$ be a real Gaussian distributed random variable $X$ with mean $\mu$ and variance $\sigma^{2}$. Then we have

$$
\mathbb{E}\left[e^{\gamma|X-\mu|}\right] \leq 2 e^{|\mu| \gamma} e^{\frac{\sigma^{2}}{2} \gamma^{2}}(\text { for all } \gamma \geq 0)
$$

and

$$
P(|X|>R) \leq \frac{2 \sigma}{\sqrt{2 \pi}(R-|\mu|)} e^{-\frac{(R-|\mu|)^{2}}{2 \sigma^{2}}}(\text { for all } R>|\mu|) .
$$

Now we present Proposition 2.1 where we give conditions which yield (i) and (ii) in Theorem 1.1. Such conditions are given in terms of the mean functions $\left\{m_{n}(\cdot): n \geq 1\right\}$ and covariance functions $\left\{k_{n}(\cdot, \cdot): n \geq 1\right\}$ of the processes $\left\{Z_{n}: n \geq 1\right\}$. 
Proposition 2.1 Condition (i) in Theorem 1.1 holds if there exist $\alpha, M_{1}, M_{2}>0$ such that

$$
\sup \left\{\frac{\left|m_{n}(t)-m_{n}(s)\right|}{|t-s|^{\alpha}}: n \geq 1, s, t \in[0,1], s \neq t\right\} \leq M_{1}
$$

and

$$
\sup \left\{v_{n} \frac{\left|k_{n}(t, t)-2 k_{n}(t, s)+k_{n}(s, s)\right|}{|t-s|^{2 \alpha}}: n \geq 1, s, t \in[0,1], s \neq t\right\} \leq M_{2} .
$$

Condition (ii) in Theorem 1.1 holds if there exists $L_{1}, L_{2}>0$ such that

$$
\limsup _{n \rightarrow \infty}\left|m_{n}(0)\right| \leq L_{1}
$$

and

$$
\limsup _{n \rightarrow \infty} v_{n} k_{n}(0,0) \leq L_{2}
$$

Remark 2.1 (On the conditions in Proposition 2.1) Conditions (8) and (10) do not depend on the speed function $v_{n}$; the constant $\alpha>0$ appears in both (8) and (9).

Proof of Proposition 2.1. For the first part of the proof we consider an arbitrarily fixed $\gamma>0$ and we take $\alpha>0$ as in (8) and (9). Then we have

$$
\begin{aligned}
\mathbb{E}\left[\exp \left(\frac{\gamma v_{n}}{|t-s|^{\alpha}}\left|Z_{n}(t)-Z_{n}(s)\right|\right)\right] \leq & 2 \exp \left(v_{n} \gamma \frac{\left|m_{n}(t)-m_{n}(s)\right|}{|t-s|^{\alpha}}\right. \\
& \left.+\frac{v_{n}^{2} \gamma^{2}}{2} \frac{\left|k_{n}(t, t)-2 k_{n}(t, s)+k_{n}(s, s)\right|}{|t-s|^{2 \alpha}}\right) \\
\leq & 2 e^{v_{n}\left(\gamma M_{1}+\frac{\gamma^{2}}{2} M_{2}\right)}
\end{aligned}
$$

by (6), (8) and (9). Thus Condition (i) in Theorem 1.1 holds; in fact we can take $n_{0}$ such that $v_{n} \geq 1$ for $n \geq n_{0}$, and $\kappa=2 e^{\gamma M_{1}+\frac{\gamma^{2}}{2} M_{2}}$.

Now the second part of the proof. For any $\delta>0$ we take $n$ large enough to have $\left|m_{n}(0)\right| \leq L_{1}+\delta$ and $v_{n} k_{n}(0,0) \leq L_{2}+\delta$. Then, for $R>L_{1}+\delta$, we have

$$
P\left(\left|Z_{n}(0)\right|>R\right) \leq \frac{2 \sqrt{k_{n}(0,0)}}{\sqrt{2 \pi}\left(R-\left(L_{1}+\delta\right)\right)} e^{-\frac{\left(R-\left(L_{1}+\delta\right)\right)^{2}}{2 k_{n}(0,0)}}
$$

by (7); therefore

$$
\begin{aligned}
\frac{1}{v_{n}} \log P\left(\left|Z_{n}(0)\right|>R\right) & \leq \frac{1}{v_{n}} \log \left(\frac{2 \sqrt{k_{n}(0,0)}}{\sqrt{2 \pi}\left(R-\left(L_{1}+\delta\right)\right)}\right)-\frac{\left(R-\left(L_{1}+\delta\right)\right)^{2}}{2 v_{n} k_{n}(0,0)} \\
& \leq \frac{1}{v_{n}} \log \left(\frac{2 \sqrt{L_{2}+\delta}}{\sqrt{2 \pi} \sqrt{v_{n}}\left(R-\left(L_{1}+\delta\right)\right)}\right)-\frac{\left(R-\left(L_{1}+\delta\right)\right)^{2}}{2\left(L_{2}+\delta\right)}
\end{aligned}
$$

and we easily get Condition (ii) in Theorem 1.1 by letting $n$ and $R$ go to infinity.

In view of what follows it is useful to check (8), (9), (10) and (11) by referring to the following conditions concerning the mean and covariance functions of the process $\{X(t): t \geq 1\}$; we remark that (13) yields (9), while (12), (14) and (15) are equivalent to (8), (10) and (11), respectively. Then we have:

$$
\sup \left\{\frac{1}{A_{n}} \sum_{k=1}^{n} a_{k} \frac{|\mu(k+t)-\mu(k+s)|}{|t-s|^{\alpha}}: n \geq 1, s, t \in[0,1], s \neq t\right\} \leq M_{1} ;
$$




$$
\sup \left\{\frac{v_{n}}{A_{n}^{2}} \frac{B_{1}^{(n)}(t, s)+B_{2}^{(n)}(t, s)+B_{3}^{(n)}(t, s)}{|t-s|^{2 \alpha}}: n \geq 1, s, t \in[0,1], s \neq t\right\} \leq M_{2},
$$

where

$$
B_{1}^{(n)}:=\sum_{k=1}^{n} a_{k}^{2} \xi(k, k, t, s), B_{2}^{(n)}:=\sum_{k=2}^{n} \sum_{h=1}^{k-1} a_{h} a_{k} \xi(k, h, t, s), B_{3}^{(n)}:=\sum_{h=2}^{n} \sum_{k=1}^{h-1} a_{h} a_{k} \xi(k, h, t, s)
$$

and

$$
\begin{gathered}
\xi(k, h, t, s):=|\varphi(k+t, k+t)-2 \varphi(k+t, k+s)+\varphi(k+s, k+s)| ; \\
\limsup _{n \rightarrow \infty} \frac{1}{A_{n}} \sum_{k=1}^{n} a_{k}|\mu(k)| \leq L_{1} ; \\
\limsup _{n \rightarrow \infty} \frac{v_{n}}{A_{n}^{2}} \sum_{k, h=1}^{n} a_{h} a_{k} \varphi(k, h) \leq L_{2} .
\end{gathered}
$$

As far as (15) is concerned, we remark that

$$
\sum_{k, h=1}^{n} a_{h} a_{k} \varphi(k, h)=\sum_{k=1}^{n} a_{k}^{2} \varphi(k, k)+2 \sum_{k=2}^{n} a_{k} \sum_{h=1}^{k-1} a_{h} \varphi(k, h) .
$$

Remark 2.2 (On (12) and (14)) As we said in Remark 2.1 for (8) and (10), conditions (12) and (14) do not depend depend on the speed function $v_{n}$. Moreover, in view of the examples presented below (see Example 3.1 in Section 3, and Section 5), note that (12) and (14) hold if the mean function $\mu(\cdot)$ is differentiable and bounded (therefore, as a particular case, if the process $\{X(t): t \geq 1\}$ is centered, i.e. $\mu(t)=0$ for all $t \geq 1)$.

\section{Results for the first class of covariance functions}

We start with a condition on the covariance function $\varphi(\cdot, \cdot)$ and we compare it with a similar condition in [11] (see Remark 3.1).

Condition 3.1 There exist two functions $f_{1}, f_{2}:[0,1] \rightarrow \mathbb{R}$ such that: $f_{1}(0)=f_{2}(0)=0, f_{1}$ and $f_{2}$ satisfy the Hölder condition, i.e. there exist $M \in(0, \infty)$ and $\gamma_{1}, \gamma_{2} \in(0,1]$ such that, for $k \in\{1,2\}$, we have

$$
\left|f_{k}(x)-f_{k}(y)\right| \leq M|x-y|^{\gamma_{k}}(\text { for all } x, y \in[0,1])
$$

and

$$
\varphi(t, s)=b_{1} f_{1}\left(q^{t+s}\right)+b_{2} f_{2}\left(q^{|t-s|}\right)(\text { for all } t, s \in[1, \infty))
$$

for some $b_{1} \in \mathbb{R}, b_{2} \geq 0$ and $q \in(0,1)$.

Remark 3.1 (Connections with hypothesis (exp) in [11]) If we consider Condition 3.1 with $f_{1}(x)=f_{2}(x)=x$ (for all $x \in[0,1]$ ), and if we also allow the case $q=0$, we recover condition (exp) in [11] by setting ( $u$ and $w$ are nonnegative constants which appear in [11])

$$
b_{1}:=u-w \text { and } b_{2}:=w .
$$

We also remark that: the continuity of the covariance function in (18) fails if we allow the case $q=0$ in Condition 3.1; if $f_{1}(x)=f_{2}(x)=x$ (for all $x \in[0,1]$ ), then we have $f_{1}(0)=f_{2}(0)=0$ and (17) (with $\gamma_{1}=\gamma_{2}=1$ ). 
Now we present the exponential tightness results. More precisely we obtain conditions (13) and (15) for some $\alpha$ and some $v_{n}$; thus, if (12) and (14) also hold (we recall that we have the same constant $\alpha$ in (12) and (13)), we have the exponential tightness of $\left\{Z_{n}: n \geq 1\right\}$ (with respect to $\left.v_{n}\right)$ by Proposition 2.1 .

Proposition 3.1 Assume that Condition 3.1 holds, and that

$$
\sup \left\{\sum_{h=1}^{k-1} a_{h} q^{\left(\gamma_{1} \wedge \gamma_{2}\right)(k-h)}: k \geq 1\right\}<\infty
$$

Then:

(i) if (3) holds, conditions (13) and (15) hold with $\alpha=\left(\gamma_{1} \wedge \gamma_{2}\right) / 2$ and $v_{n}=A_{n}$;

(ii) if (4) holds, conditions (13) and (15) hold with $\alpha=\left(\gamma_{1} \wedge \gamma_{2}\right) / 2$ and $v_{n}=A_{n}^{2}$.

Proof. We start with the proof of (13). Firstly, by (18) and after some manipulations with the triangle inequality, we have

$$
\begin{aligned}
\mid \varphi(k+t, h+t)- & 2 \varphi(k+t, h+s)+\varphi(k+s, h+s) \mid \\
= & \mid b_{1} f_{1}\left(q^{k+h+2 t}\right)+b_{2} f_{2}\left(q^{|k-h|}\right)-2\left(b_{1} f_{1}\left(q^{k+h+t+s}\right)+b_{2} f_{2}\left(q^{|k+t-(h+s)|}\right)\right) \\
& +b_{1} f_{1}\left(q^{k+h+2 s}\right)+b_{2} f_{2}\left(q^{|k-h|}\right) \mid \\
\leq & b_{1} \mid\left(\left|f_{1}\left(q^{k+h+2 t}\right)-f_{1}\left(q^{k+h+t+s}\right)\right|+\left|f_{1}\left(q^{k+h+t+s}\right)-f_{1}\left(q^{k+h+2 s}\right)\right|\right) \\
& +2 b_{2}\left|f_{2}\left(q^{|k-h|}\right)-f_{2}\left(q^{|k+t-(h+s)|}\right)\right| ;
\end{aligned}
$$

therefore, by (17) and after some computations, we obtain

$$
\begin{aligned}
\mid \varphi(k+t, h+t) & -2 \varphi(k+t, h+s)+\varphi(k+s, h+s) \mid \\
= & \left|b_{1}\right| M q^{\gamma_{1}(k+h+t+s)}\left(\left|q^{t-s}-1\right|^{\gamma_{1}}+\left|1-q^{s-t}\right|^{\gamma_{1}}\right)+2 b_{2} M\left|q^{|k-h|}-q^{|k+t-(h+s)|}\right|^{\gamma_{2}} .
\end{aligned}
$$

Moreover we remark that $q^{\gamma_{1}(k+h+t+s)} \leq q^{\gamma_{1}|k-h|}$ and

$$
\left|q^{|k-h|}-q^{|k+t-(h+s)|}\right|^{\gamma_{2}}=q^{\gamma_{2}|k-h|}\left|1-q^{|k+t-(h+s)|-|k-h|}\right|^{\gamma_{2}}
$$

where

$$
|k+t-(h+s)|-|k-h|= \begin{cases}|t-s| & \text { if } k=h \\ t-s & \text { if } k>h \\ s-t & \text { if } k<h\end{cases}
$$

Therefore condition (13) can be easily checked with $\alpha=\left(\gamma_{1} \wedge \gamma_{2}\right) / 2$ if we show that

$$
\sup \left\{\frac{v_{n}}{A_{n}^{2}} \sum_{k, h=1}^{n} a_{k} a_{h} q^{\gamma_{i}|k-h|}: n \geq 1\right\}<\infty(\text { for } i \in\{1,2\})
$$

to this aim it is enough to check that

$$
\sup \left\{\frac{v_{n}}{A_{n}^{2}} \sum_{k=1}^{n} a_{k}^{2}: n \geq 1\right\}<\infty \text { and } \sup \left\{\frac{v_{n}}{A_{n}^{2}} \sum_{k=2}^{n} a_{k} \sum_{h=1}^{k-1} a_{h} q^{\left(\gamma_{1} \wedge \gamma_{2}\right)(k-h)}: n \geq 1\right\}<\infty .
$$

Now we distinguish the cases (i) and (ii). In the first case (where we have $v_{n}=A_{n}$ ) the first quantity is finite by taking into account (3), and the second one is finite by taking into account (19). In the second case (where we have $v_{n}=A_{n}^{2}$ ) the first quantity is finite by taking into account (4) and the finiteness of $\sum_{k=1}^{\infty} \frac{1}{k^{2}}$, and the second one is finite by (17) in [11]. 
We conclude with the proof of (15). Firstly we have

$$
|\varphi(k, h)| \leq\left|b_{1}\right| M q^{\gamma_{1}(k+h)}+b_{2} M q^{\gamma_{2}|k-h|} \leq\left(\left|b_{1}\right|+b_{2}\right) M q^{\left(\gamma_{1} \wedge \gamma_{2}\right)|k-h|}
$$

with some standard manipulations where we take into account (18), $f_{1}(0)=f_{2}(0)=0, k+h \geq|k-h|$ and (17). Then, if we set $\tilde{M}:=\left(\left|b_{1}\right|+b_{2}\right) M$, by (16) we have

$$
\sum_{k, h=1}^{n} a_{h} a_{k} \varphi(k, h) \leq \tilde{M} \sum_{k=1}^{n} a_{k}^{2}+2 \tilde{M} \sum_{k=2}^{n} a_{k} \sum_{h=1}^{k-1} a_{h} q^{\left(\gamma_{1} \wedge \gamma_{2}\right)(k-h)} .
$$

Therefore condition (15) can be easily checked if we show that

$$
\limsup _{n \rightarrow \infty} \frac{v_{n}}{A_{n}^{2}} \sum_{k=1}^{n} a_{k}^{2}<\infty \text { and } \limsup _{n \rightarrow \infty} \frac{v_{n}}{A_{n}^{2}} \sum_{k=2}^{n} a_{k} \sum_{h=1}^{k-1} a_{h} q^{\left(\gamma_{1} \wedge \gamma_{2}\right)(k-h)}<\infty .
$$

These two conditions hold (under both conditions (i) and (ii)) because we already show that (20) hold.

Remark 3.2 Now we briefly discuss Propositions 3.1 and 3.2(i) in [11], i.e. the proof of the limit (5), in terms of Condition 3.1. The proofs can be repeated under some slightly different hypotheses: $f_{1}(x)=f_{2}(x)=x$ (for all $x \in[0,1]$ ) which yields $f_{1}(0)=f_{2}(0)=0$; the case $q=0$ can be allowed. The propositions (and their proofs) could be repeated here with suitable modifications; for instance we have the constants $b_{1}$ and $b_{2}$ in place of $u$ and $w$ (see Remark 3.1). The details are omitted.

We conclude this section with Example 3.1; another example concerning Condition 3.1 is presented in Section 5 (Example 5.6). Example 3.1 is a revised version of the example in [11] based on the classical Ornstein-Uhlenbeck process with affine drift; the revised version of the example in this paper takes into account Condition 3.1 (we recall Remark 3.1 with the connections between Condition 3.1 in this paper and hypothesis (exp) in [11]).

Example 3.1 (Classical Ornstein-Uhlenbeck process with affine drift) Let $\{X(t): t \geq 0\}$ be the solution of the stochastic differential equation

$$
d X(t)=(-\alpha X(t)+\beta) d t+\sigma d B(t)
$$

where $\alpha, \sigma>0, \beta \in \mathbb{R},\{B(t): t \geq 0\}$ is a standard Brownian motion, and $X(0)$ is a Normal distributed random variable with mean $\mu_{0}$ and variance $\sigma_{0}^{2}$. It is known that, for $0 \leq s \leq t$, we have

$$
\varphi(t, s):=e^{-\alpha(t-s)}\left(\sigma_{0}^{2} e^{-2 \alpha s}+\frac{\sigma^{2}}{2 \alpha}\left(1-e^{-2 \alpha s}\right)\right)=\left(\sigma_{0}^{2}-\frac{\sigma^{2}}{2 \alpha}\right) e^{-\alpha(t+s)}+\frac{\sigma^{2}}{2 \alpha} e^{-\alpha(t-s)} .
$$

Thus Condition 3.1 holds with $b_{1}=\sigma_{0}^{2}-\frac{\sigma^{2}}{2 \alpha}, b_{2}=\frac{\sigma^{2}}{2 \alpha}, q=e^{-\alpha}$, and $f_{1}(x)=f_{2}(x)=x$ for all $x \in[0,1]$ (and therefore $\gamma_{1}=\gamma_{2}=1$ as pointed out in Remark 3.1). Finally we notice that, in connection with Remark 2.2, we have

$$
\mu(t)=e^{-\alpha t} \mu_{0}+\frac{\beta}{\alpha}\left(1-e^{-\alpha t}\right)(\text { for all } t \geq 0) .
$$

\section{Results for the second class of covariance functions}

We start with a condition on the covariance function $\varphi(\cdot, \cdot)$ and we compare it with a similar condition in [11] (see Remark 4.1). 
Condition 4.1 There exist a function $g:[0,1] \rightarrow[0, \infty)$ such that: $g(0)=0, g$ satisfies the Hölder condition, i.e. there exist $M \in(0, \infty)$ and $\gamma \in(0,1]$ such that

$$
|g(x)-g(y)| \leq M|x-y|^{\gamma}(\text { for all } x, y \in[0,1])
$$

and

$$
\varphi(t, s)=g\left(\frac{s \wedge t}{s \vee t}\right) \quad(\text { for all } t, s \in[1, \infty))
$$

Remark 4.1 (Connections with hypothesis (power) in [11]) If we consider Condition 4.1 with

$$
g(x) \leq M x^{\gamma}(\text { for all } x \in[0,1])
$$

in place of (21) (note that (21) yields (23)), we recover condition (power) in [11] by setting ( $f, c$ and $\rho$ are items in [11])

$$
g(x):=\frac{f(x)}{x^{c}} \text { and } \gamma:=1-\rho .
$$

Actually here we also allow the case $\rho=0$ which was neglected in [11].

Now we present the exponential tightness result. As in Proposition 3.1, we obtain conditions (13) and (15) for some $\alpha$ and some $v_{n}$; thus, if (12) and (14) also hold (we recall that we have the same constant $\alpha$ in (12) and (13)), we have the exponential tightness of $\left\{Z_{n}: n \geq 1\right\}$ (with respect to $v_{n}$ ) by Proposition 2.1 .

Proposition 4.1 Assume that Condition 4.1 holds. Then, if (4) holds, conditions (13) and (15) hold with $\alpha=\gamma / 2$ and $v_{n}=A_{n}$.

Proof. We start with the proof of (13) with $\alpha=\gamma / 2$ and $v_{n}=A_{n}$. It suffices to show that

$$
S_{j}:=\sup \left\{\frac{1}{A_{n}} \frac{B_{j}^{(n)}(t, s)}{|t-s|^{\gamma}}: n \geq 1, s, t \in[0,1], s \neq t\right\}(\text { for } j \in\{1,2,3\})
$$

are finite.

We start with the case $j=1$, i.e. $k=h$; moreover for the moment we assume that $t \geq s$. Then, by taking into account Condition 4.1, we have

$$
\begin{aligned}
\mid \varphi(k+t, k+t) & -2 \varphi(k+t, k+s)+\varphi(k+s, k+s) \mid \\
= & \left|g(1)-2 g\left(\frac{k+s}{k+t}\right)+g(1)\right|=2\left|g(1)-g\left(\frac{k+s}{k+t}\right)\right| \\
\leq & 2 M\left|1-\frac{k+s}{k+t}\right|^{\gamma} \leq 2 M \frac{(t-s)^{\gamma}}{k^{\gamma}} .
\end{aligned}
$$

Then, for all $t, s \in[0,1]$, we have

$$
|\varphi(k+t, k+t)-2 \varphi(k+t, k+s)+\varphi(k+s, k+s)| \leq 2 M \frac{|t-s|^{\gamma}}{k^{\gamma}}
$$

thus, by also taking into account (4), we complete the proof for the case $j=1$ noting that

$$
S_{1} \leq 2 M \sup \left\{\frac{1}{A_{n}} \sum_{k=1}^{n} a_{k}^{2} \cdot \frac{1}{k^{\gamma}}: n \geq 1\right\} \leq 2 C^{2} M \sup \left\{\frac{1}{A_{n}} \sum_{k=1}^{n} \frac{1}{k^{2+\gamma}}: n \geq 1\right\}<\infty .
$$


Now we consider the case $j=2$, i.e. $k>h$. Then, by taking into account Condition 4.1, for all $t, s \in[0,1]$ we have

$$
\begin{aligned}
& \mid \varphi(k+t, h+t)-2 \varphi(k+t, h+s)+\varphi(k+s, h+s) \mid \\
&=\left|g\left(\frac{h+t}{k+t}\right)-2 g\left(\frac{h+s}{k+t}\right)+g\left(\frac{h+s}{k+s}\right)\right| \\
& \leq\left|g\left(\frac{h+t}{k+t}\right)-g\left(\frac{h+s}{k+t}\right)\right|+\left|g\left(\frac{h+s}{k+s}\right)-g\left(\frac{h+s}{k+t}\right)\right| \\
& \leq M \frac{|t-s|^{\gamma}}{(k+t)^{\gamma}}+M(h+s)^{\gamma} \frac{|t-s|^{\gamma}}{(k+t)^{\gamma}(k+s)^{\gamma}} \\
& \leq M|t-s|^{\gamma}\left(\frac{1}{(k+t)^{\gamma}}+\frac{1}{(k+t)^{\gamma}}\right) \leq 2 M \frac{|t-s|^{\gamma}}{k^{\gamma}} .
\end{aligned}
$$

thus, by also taking into account (4), we complete the proof for the case $j=2$ noting that

$$
\begin{aligned}
S_{2} & \leq 2 M \sup \left\{\frac{1}{A_{n}} \sum_{k=2}^{n} a_{k} \sum_{h=1}^{k-1} a_{h} \frac{1}{k^{\gamma}}: n \geq 1\right\} \leq 2 C^{2} M \sup \left\{\frac{1}{A_{n}} \sum_{k=2}^{n} \frac{1}{k^{1+\gamma}} \sum_{h=1}^{k-1} \frac{1}{h}: n \geq 1\right\} \\
& \leq 2 C^{2} M \sup \left\{\frac{1}{A_{n}} \sum_{k=2}^{n} \frac{1}{k^{1+\gamma}}(1+\log (k-1)): n \geq 1\right\}<\infty .
\end{aligned}
$$

The proof for $j=3$, i.e. the proof of the finiteness of $S_{3}$, is similar to the one for $j=2$ (by exchanging the role of $h$ and $k$ because we have $k<h$ instead of $k>h$ ).

We conclude with the proof of (15) with $v_{n}=A_{n}$. Firstly we have

$$
\sum_{k, h=1}^{n} a_{h} a_{k} \varphi(k, h)=g(1) \sum_{k=1}^{n} a_{k}^{2}+2 \sum_{k=2}^{n} a_{k} \sum_{h=1}^{k-1} a_{h} g(h / k)
$$

by (16). Then, by (4) and by Condition 4.1 (in particular here we take into account that $g(0)=0$ ), we have

$$
\sum_{k, h=1}^{n} a_{h} a_{k} \varphi(k, h) \leq g(1) C^{2} \sum_{k=1}^{n} \frac{1}{k^{2}}+2 C M \sum_{k=2}^{n} a_{k} \sum_{h=1}^{k-1} \frac{1}{h}\left(\frac{h}{k}\right)^{\gamma} .
$$

Moreover we remark that

$$
\begin{aligned}
\sum_{k=2}^{n} a_{k} \sum_{h=1}^{k-1} \frac{1}{h}\left(\frac{h}{k}\right)^{\gamma} & =\sum_{k=2}^{n} \frac{a_{k}}{k^{\gamma}} \sum_{h=1}^{k-1} \frac{1}{h^{1-\gamma}} \leq \sum_{k=2}^{n} \frac{a_{k}}{k^{\gamma}} \int_{0}^{k-1} \frac{1}{x^{1-\gamma}} d x \\
& =\sum_{k=2}^{n} \frac{a_{k}}{k^{\gamma}} \cdot \frac{(k-1)^{\gamma}}{\gamma} \leq \frac{1}{\gamma} \sum_{k=2}^{n} a_{k} \leq \frac{A_{n}}{\gamma} ;
\end{aligned}
$$

then

$$
\frac{1}{A_{n}} \sum_{k, h=1}^{n} a_{h} a_{k} \varphi(k, h) \leq \frac{g(1) C^{2}}{A_{n}} \sum_{k=1}^{n} \frac{1}{k^{2}}+\frac{2 C M}{A_{n}} \cdot \frac{A_{n}}{\gamma}=\frac{g(1) C^{2}}{A_{n}} \sum_{k=1}^{n} \frac{1}{k^{2}}+\frac{2 C M}{\gamma}
$$

and we complete the proof by taking into account (2) and the finiteness of $\sum_{k=1}^{\infty} \frac{1}{k^{2}}$.

Remark 4.2 Now we briefly discuss Proposition 3.3 in [11], i.e. the proof of the limit (5), in terms of Condition 4.1. We remark that this limit can be proved under some slightly different hypotheses: we can consider (23) in place of (21). In connection with Remark 4.1, we can say that the proof of Proposition 3.3 in [11] still works with $x \mapsto g(x)$ and $1-\gamma$ in this paper in place of $x \mapsto \frac{f(x)}{x^{c}}$ and $\rho \in(0,1)$ in [11] (in particular we have $c(t, s):=2 \tilde{\ell} \int_{0}^{1} \frac{g(x)}{x} d x$, for all $t, s \in[0,1]$, 
where $\tilde{\ell}:=\lim _{n \rightarrow \infty} n a_{n}$ as in (13) in [11]); moreover here we allow the case $\gamma=1$ while the case $\rho=0$ was neglected in [11] (see condition (power) in that reference), and this is possible with suitable minor modifications of some parts of the proof (in some cases the steps of the proof are even easier). The details are omitted.

\section{$5 \quad$ Examples}

In Section 3 we presented Example 3.1 concerning Condition 3.1. In this section we present several examples of self-similar process such that Condition 4.1 holds; finally we present an example concerning Condition 3.1 where we have Lamperti's transforms of normalized self-similar processes which satisfy Condition 4.1.

In all the examples presented in this section (except the final Example 5.6) we assume that $\{X(t): t \geq 1\}$ is defined by

$$
X(t):=\frac{V(t)}{t^{H}}(\text { for all } t \geq 1),
$$

where $\{V(t): t \geq 0\}$ is a centered self-similar Gaussian process with parameter $H>0$. We recall that the law of the process $\{V(t): t \geq 0\}$ is determined by its covariance function, which satisfies the following condition (see e.g. Example 8.9.2 in [6])

$$
\left.\operatorname{Cov}(V(t), V(s))=t^{2 H} \operatorname{Cov}(V(1), V(s / t)) \text { (for all } s \geq 0 \text { and } t>0\right) ;
$$

then, since we are interested in Condition 4.1, we can easily check that

$$
\varphi(t, s)=\frac{1}{t^{H} s^{H}} \operatorname{Cov}(V(t), V(s))=\frac{\operatorname{Cov}(V(1), V(s / t))}{(s / t)^{H}}(\text { for all } t \geq s \geq 1),
$$

and we can say that (22) holds with

$$
g(x):=x^{-H} \operatorname{Cov}(V(1), V(x)) .
$$

For each one of the examples below (except the final Example 5.6) we can say that (24) holds for a specific value of $H$ (in several cases this is well-known) and we check that $g$ is a nonnegative function (defined on $[0,1]$ ) which satisfies the Hölder condition (21) for a suitable $\gamma \in(0,1]$, and $g(0)=0$.

We recall that in some cases we have revised versions of the examples in [11] (Example 5.1 with $k=1$ and Example 5.4); these revisions take into account Condition 4.1 in this paper (we recall Remark 4.1 with the connections between Condition 4.1 in this paper and hypothesis (power) in [11]).

We start with Examples 5.1, 5.2 and 5.3, in which we have the following common feature: the Hölder condition (21) for the function $g$ holds with $\gamma:=\gamma_{(0)} \wedge \gamma_{(1)}$, where $\gamma_{(0)}$ and $\gamma_{(1)}$ are two Hölder exponents which depend on the behavior of the function $g$ at $x=0$ and at $x=1$ (in fact the restriction of $g$ on $(0,1)$ is a regular function).

Example 5.1 (Bifractional Brownian Motion) We have

$$
\operatorname{Cov}(V(t), V(s))=\frac{\sigma^{2}}{2^{k}}\left\{\left(t^{2 h}+s^{2 h}\right)^{k}-|t-s|^{2 h k}\right\}(\text { for all } t, s \geq 0)
$$

for $\sigma^{2}>0, h \in(0,1)$ and $k \in(0,2)$ such that $h k \in(0,1)$ (see e.g. [15], Definition 1.1). Then (24) holds with $H=h k$, and the function $g$ in (25) is

$$
g(x):=\frac{\sigma^{2}}{2^{k}} x^{-h k}\left\{\left(1+x^{2 h}\right)^{k}-(1-x)^{2 h k}\right\} .
$$


One can easily check that $g$ is nonnegative and $\gamma_{(1)}=(2 h k) \wedge 1$. Moreover $\gamma_{(0)}=(h(2-k)) \wedge(1-h k)$ because, near $x=0$, the function $g$ behaves like

$$
\frac{\sigma^{2}}{2^{k}} x^{-h k}\left\{\left(1+k x^{2 h}\right)-(1-2 h k x)\right\}=\frac{\sigma^{2}}{2^{k}}\left\{k x^{2 h-h k}+2 h k x^{1-h k}\right\}
$$

(note that this allows to say that $g(0)=0)$. In conclusion we have $\gamma=\gamma_{(0)} \wedge \gamma_{(1)}=(h(2-k)) \wedge$ $(1-h k) \wedge(2 h k)$.

Example 5.2 (Sub-fractional Brownian Motion) We have

$$
\operatorname{Cov}(V(t), V(s))=\frac{\sigma^{2}}{2-2^{2 h-1}}\left\{t^{2 h}+s^{2 h}-\frac{1}{2}\left((s+t)^{2 h}+|t-s|^{2 h}\right)\right\}(\text { for all } t, s \geq 0)
$$

for $\sigma^{2}>0$ and $h \in(0,1)$ (see e.g. [15], Definition 1.1). Then (24) holds with $H=h$, and the function $g$ in $(25)$ is

$$
\begin{aligned}
g(x):=\frac{\sigma^{2}}{2-2^{2 h-1}} x^{-h}\left\{1+x^{2 h}-\frac{1}{2}\left((1+x)^{2 h}+(1-x)^{2 h}\right)\right\} & \\
& =\frac{\sigma^{2}}{2\left(2-2^{2 h-1}\right)} x^{-h}\left\{2\left(1+x^{2 h}\right)-(1+x)^{2 h}-(1-x)^{2 h}\right\} .
\end{aligned}
$$

One can easily check that $g$ is nonnegative (namely one can check that $\frac{1}{2}\left((1+x)^{2 h}+(1-x)^{2 h}\right) \leq 1$ ) and $\gamma_{(1)}=(2 h) \wedge 1$. Moreover $\gamma_{(0)}=h$ because, near $x=0$, the function $g$ behaves like

$$
\frac{\sigma^{2}}{2\left(2-2^{2 h-1}\right)} x^{-h}\left\{2\left(1+x^{2 h}\right)-(1+2 h x)-(1-2 h x)\right\}=\frac{\sigma^{2} x^{h}}{\left(2-2^{2 h-1}\right)}
$$

(note that this allows to say that $g(0)=0$ ). In conclusion we have $\gamma=\gamma_{(0)} \wedge \gamma_{(1)}=h$.

Example 5.3 (Another example cited in [15]) We have

$$
\operatorname{Cov}(V(t), V(s))=\left\{\begin{array}{ll}
\frac{\Gamma(1-K)}{K}\left\{t^{K}+s^{K}-(t+s)^{K}\right\} & \text { if } K \in(0,1) \\
\frac{\Gamma(2-K)}{K(K-1)}\left\{(t+s)^{K}-t^{K}-s^{K}\right\} & \text { if } K \in(1,2)
\end{array} \quad \text { (for all } t, s \geq 0\right)
$$

(see e.g. [15], equation (2.1) and the successive displayed formula; see also [14] cited therein). Then (24) holds with $H=K / 2$, and the function $g$ in (25) is

$$
g(x):=C_{K} x^{-K / 2}\left(1+x^{K}-(1+x)^{K}\right)
$$

for a suitable positive constant $C_{K}$ (which depends on $K$ ). One can check that $g$ is nonnegative (namely one can check that the function $1+x^{K}-(1+x)^{K}$ is increasing in $[0,1]$ ) and, since the behavior of the function $g$ near $x=1$ is regular, we have $\gamma_{(1)}=1$. Moreover $\gamma_{(0)}=(K / 2) \wedge(1-K / 2)$ because, near $x=0$, the function $g$ behaves like

$$
C_{K} x^{-K / 2}\left\{1+x^{K}-(1+K x)\right\}=C_{K} x^{K / 2}-K x^{1-K / 2}
$$

(note that this allows to say that $g(0)=0$ ). In conclusion we have $\gamma=\gamma_{(0)} \wedge \gamma_{(1)}=(K / 2) \wedge(1-K / 2)$.

We have two further examples concerning Condition 4.1.

Example 5.4 (Integrated Brownian Motion) We have

$$
\begin{aligned}
\operatorname{Cov}(V(t), V(s)) & =\frac{1}{(a !)^{2}} \int_{0}^{\sigma^{2} \cdot s \wedge t}\left(\sigma^{2} s-r\right)^{a}\left(\sigma^{2} t-r\right)^{a} d r \\
& =\frac{\sigma^{4 a+2}}{(a !)^{2}} \int_{0}^{s \wedge t}(s-u)^{a}(t-u)^{a} d u(\text { for all } t, s \geq 0)
\end{aligned}
$$


for $\sigma^{2}>0$ and $a \in\{0,1,2, \ldots\}$ (see e.g. [2] and the references cited therein; the process $\{V(t): t \geq$ $0\}$ is the a-th fold Integrated Brownian Motion with variance parameter $\sigma^{2}>0$, and $a=0$ means no integration). Then (24) holds with $H=a+\frac{1}{2}$, and the function $g$ in (25) is

$$
g(x):=\frac{\sigma^{4 a+2}}{(a !)^{2}} \cdot x^{1 / 2} \cdot \int_{0}^{1}(1-y)^{a}(1-x y)^{a} d y
$$

(this can be checked after some easy computations). Finally we can say that the function $g$ is nonnegative, $g(0)=0$ and $\gamma=\frac{1}{2}$.

Example 5.5 (Gaussian Martingale) We have

$$
\operatorname{Cov}(V(t), V(s))=\frac{(s \wedge t)^{2 \eta+1}}{2 \eta+1}(\text { for all } t, s \geq 0)
$$

for some $\eta \geq 0$ (see e.g. [7], Section 4.1; the process $\{V(t): t \geq 0\}$ is defined by $V(t):=\int_{0}^{t} t^{\eta} d B(t)$, where $\{B(t): t \geq 0\}$ is a standard Brownian Motion). Then (24) holds with $H=\eta+\frac{1}{2}$, and the function $g$ in $(25)$ is

$$
g(x):=\frac{x^{\eta+1 / 2}}{2 \eta+1}
$$

Finally we can say that the function $g$ is nonnegative, $g(0)=0$ and $\gamma=(\eta+1 / 2) \wedge 1$.

We conclude with Example 5.6 concerning Condition 3.1. In view of this we consider a process $\{Y(t): t>0\}$ defined by

$$
Y(t):=\frac{V(t)}{t^{H}}(\text { for all } t \geq 0)
$$

where $\{V(t): t \geq 0\}$ is a self-similar process with parameter $H>0$ which satisfies Condition 4.1. Then the process $\{X(t): t \geq 1\}$ will be the Lamperti's transform (also called Doob's transform) of $\{Y(t): t>0\}$. As a particular case of Example 5.6 we have the fractional Ornstein-Uhlenbeck process of the second kind (see e.g. Section 3 in [12]). On the contrary we believe that the fractional Ornstein-Uhlenbeck process of the first kind (which is the unique strong solution of the Langevin stochastic differential equation with fractional Brownian motion noise; see e.g. Section 2.2 in [12]) cannot be considered as an example which satisfies Condition 3.1 or Condition 4.1. For completeness we cite [3] as an important reference where these two fractional Ornstein-Uhlenbeck processes are studied.

Example 5.6 (Lamperti's transform of normalized self-similar processes) $\operatorname{Let}\{Y(t): t>$ $0\}$ be defined as above. Then, for $\alpha>0$, its Lamperti's transform is defined by

$$
X(t):=e^{-\alpha t} \cdot Y\left(\frac{H}{\alpha} \cdot e^{\alpha t / H}\right) .
$$

So we have

$$
X(t)=e^{-\alpha t}\left(\frac{H}{\alpha} \cdot e^{\alpha t / H}\right)^{H} \cdot V\left(\frac{H}{\alpha} \cdot e^{\alpha t / H}\right)=\left(\frac{H}{\alpha}\right)^{H} \cdot V\left(\frac{H}{\alpha} \cdot e^{\alpha t / H}\right)
$$

and, if we consider the function $g$ concerning the covariance function of the process $\{V(t): t \geq 0\}$ (we recall that it satisfies Condition 4.1), we get

$$
\varphi(t, s)=\left(\frac{H}{\alpha}\right)^{2 H} g\left(e^{-\alpha(t-s) / H}\right)(\text { for all } t \geq s \geq 1)
$$


after some easy computations. In conclusion Condition 3.1 holds; namely we have (18) with

$$
b_{1}=0, b_{2}=\left(\frac{H}{\alpha}\right)^{2 H}, f_{2}=g \text { and } q=e^{-\alpha / H}
$$

(thus $\left.\gamma_{2}=\gamma\right)$.

As a particular case we can consider the fractional Ornstein-Uhlenbeck process of the second kind, i.e. the Lamperti's transform of the fractional Brownian Motion $\{V(t): t \geq 0\}$ as in Example 5.1 with $h=H$ and $k=1$; thus Condition 3.1 holds with the positions above, where $f_{2}$ and $\gamma_{2}$ coincide with $g$ and $\gamma$ in Example 5.1 with $h=H$ and $k=1$ (thus $\gamma=H \wedge 1-H$ ).

We remark that, in connection with Remark 2.2, in all the examples in this section we have $\mu(t)=0$ for all $t \geq 0$.

\section{A Proof of Lemma 2.1}

We start with the proof of (6). The moment generating function of the random variable $X$ is

$$
\mathbb{E}\left[e^{\theta X}\right]=e^{\mu \theta+\frac{\sigma^{2}}{2} \theta^{2}}(\text { for all } \theta \in \mathbb{R}) .
$$

Then, for all $\eta \geq 0$, we have

$$
\mathbb{E}\left[e^{\eta X}\right] \leq e^{|\mu| \eta} \mathbb{E}\left[e^{\eta|X-\mu|}\right]
$$

and

$$
\mathbb{E}\left[e^{\eta|X-\mu|}\right]=\mathbb{E}\left[e^{\eta(X-\mu)} 1_{X-\mu \geq 0}\right]+\mathbb{E}\left[e^{\eta(\mu-X)} 1_{X-\mu<0}\right]=2 \mathbb{E}\left[e^{\eta(X-\mu)} 1_{X-\mu \geq 0}\right]
$$

(the last equality holds because $X-\mu$ and $\mu-X$ are equally distributed); thus we get (6) by putting the pieces together, and by considering the moment generating function of the centered Gaussian distributed random variables $X-\mu$.

Now the proof of (7). Let $\bar{\Phi}$ be the function defined by

$$
\bar{\Phi}(x):=\frac{1}{\sqrt{2 \pi}} \int_{x}^{\infty} e^{-\frac{y^{2}}{2}} d y .
$$

Then, if $R>|\mu|$, or equivalently $R-\mu>0$ and $R+\mu>0$, we have

$$
\begin{aligned}
P(|X|>R) & =P(X>R)+P(X<-R)=\bar{\Phi}\left(\frac{R-\mu}{\sigma}\right)+1-\bar{\Phi}\left(\frac{-R-\mu}{\sigma}\right) \\
& =\bar{\Phi}\left(\frac{R-\mu}{\sigma}\right)+\bar{\Phi}\left(\frac{R+\mu}{\sigma}\right)
\end{aligned}
$$

and, by the well-known estimate

$$
\bar{\Phi}(x) \leq \frac{1}{\sqrt{2 \pi} x} e^{-\frac{x^{2}}{2}}(\text { for all } x>0)
$$

(see e.g. [8], Chapter 7, Lemma 2, Equation (1.8)), we obtain

$$
P(|X|>R) \leq \frac{\sigma}{\sqrt{2 \pi}(R-\mu)} e^{-\frac{(R-\mu)^{2}}{2 \sigma^{2}}}+\frac{\sigma}{\sqrt{2 \pi}(R+\mu)} e^{-\frac{(R+\mu)^{2}}{2 \sigma^{2}}} .
$$

In conclusion (7) is proved. We remark that (7) trivially holds even if $\sigma^{2}=0$, or equivalently $X=\mu$ almost surely (in fact we have $P(|X|>R)=0$ for $R>|\mu|$ ). 


\section{Acknowledgements}

The authors thank the referee for several hints and comments which led to a great improvement of the paper (in particular the presentation of the examples). The support of Gruppo Nazionale per l'Analisi Matematica, la Probabilità e le loro Applicazioni (GNAMPA) of the Istituto Nazionale di Alta Matematica (INdAM) is acknowledged.

\section{References}

[1] G.A. Brosamler. An almost everywhere central limit theorem. Math. Proc. Camb. Phil. Soc. 104 (1988) 561-574.

[2] X. Chen, W.V. Li. Quadratic functionals and small ball probabilities for the $m$-fold integrated Brownian motion. Ann. Probab. 31 (2003) 1052-1077.

[3] P. Cheridito, H. Kawaguchi, M. Maejima. Fractional Ornstein-Uhlenbeck processes. Electron. J. Probab. 8 (2003) paper no. 3, 14 pp.

[4] A. Dembo, O. Zeitouni. Large Deviations Techniques and Applications. 2nd. Edition. Springer, 1998.

[5] B. Djehiche, A. Schied. Large deviations for hierarchical systems of interacting jump processes. J. Theoret. Probab. 11 (1998) 1-24.

[6] P. Embrechts, C. Klüppelberg, T. Mikosch. Modelling Extremal Events. Springer, 1997.

[7] J.Y. Fan, K. Hamza, F. Klebaner. Mimicking self-similar processes. Bernoulli 21 (2015) 13411360 .

[8] W. Feller. An Introduction to Probability Theory and its Applications. Volume I. 3rd. Edition. John Wiley and Sons, 1968.

[9] A. Fisher. Convex-invariant means and a pathwise central limit theorem. Adv. in Math. 63 (1987) 213-246.

[10] A. Fisher. A pathwise central limit theorem for random walk. Preprint (1989).

[11] R. Giuliano, C. Macci, B. Pacchiarotti. On large deviations for some sequences of weighted means of Gaussian processes. J. Math. Anal. Appl. 414 (2014) 612-631.

[12] T. Kaarakka, P. Salminen. On fractional Ornstein-Uhlenbeck processes. Commun. Stoch. Anal. 5 (2011) 121-133.

[13] M.T. Lacey and W. Philipp. A note on the almost everywhere central limit theorem. Statist. Probab. Lett. 9 (1990) 201-205.

[14] P. Lei, D. Nualart. A decomposition of the bi-fractional Brownian motion and some applications. Statist. Probab. Lett. 79 (2009) 619-624.

[15] M. Matsui, N.-R. Shieh. The Lamperti transforms of self-similar Gaussian processes and their exponentials. Stoch. Models 30 (2014) 68-98.

[16] P. Schatte. On strong versions of the central limit theorem. Math. Nachr. 137 (1988) 249-256.

[17] A. Schied. Criteria for exponential tightness in path spaces. Discussion Paper, SFB 303, Bonn (1995). Available at http://aschied.de/expo.pdf

[18] A. Schied. Moderate deviations and functional LIL for super-Brownian motion. Stochastic Process. Appl. 72 (1997) 11-25. 